\title{
Incontinencia Pigmenti. Presentación de un Caso.
}

\author{
Pigmenti incontinence. Presentation of a case.
}

*Alejandra Aldana Raudales, *Dulce María Corea, **Héctor Rubén Caballero Castro.

\section{RESUMEN}

La incontinentia pigmenti es una genodermatosis que afecta la pigmentación de la piel y suele estar asociada con una gran variedad de alteraciones en ojos, uñas, pelo, dientes, esqueleto, corazón y sistema nervioso central. La mayoría de los casos de incontinencia pigmenti se producen en forma esporádica como resultado de una mutación de novo consistente en la deleción de gran parte del gen NEMO (Nuclear Factor Kappa B Essential Modifier).

Se presenta una paciente de 6 años de edad con lesiones hiperpigmentadas siguiendo las líneas de Blaschko. No tiene antecedentes durante el periodo perinatal, nació vía vaginal sin complicaciones, con un desarrollo cognitivo normal para su edad, sin afectación neurológica, lo cual llama la atención pues aunque esta enfermedad afecta principalmente a la piel, hay que considerar otros trastornos asociados, incluyendo defectos dentales, episodios convulsivos, retraso mental, anomalías oculares y neoplasias infantiles.

\section{PALABRAS CLAVES}

Incontinencia pigmenti, informes de casos.

\section{ABSTRACT}

The incontinentia pigmenti is a genodermatosis that affects the pigmentation of the skin and is usually associated with a wide variety of alterations in eyes, nails, hair, teeth, skeleton, heart and central nervous system. Most cases

* Médico Residente de Pediatría III año UNAH-VS.

**Dermatólogo Pediatra, IHSS.

Dirigir correspondencia a: alealdana286@hotmail.com

Recibido: 27 de julio 2017 Aprobado: 12 de septiembre 2017 of pigmenti incontinence occur sporadically as a result of a de novo mutation consisting of the deletion of a large part of the NEMO gene (Nuclear Factor Kappa B Essential Modifier).

We present a 6-year-old patient with hyperpigmented lesions following the Blaschko lines. She has no history during the perinatal period, was born vaginally without complications, with a normal cognitive development for her age, without neurological affectation, which calls attention because although this disease mainly affects the skin, other associated disorders must be considered, including dental defects, convulsive episodes, mental retardation, ocular abnormalities and childhood neoplasms.

\section{KEYWORDS}

Pigment incontinence, case reports.

\section{INTRODUCCIÓN}

La incontinencia pigmenti (IP), incontinencia pigmentaria o síndrome de Bloch-Sulzberger es una genodermatosis rara con un patrón de herencia ligado al cromosoma X. Fue descrita por Bloch y Sulzberger en 1926 y $1928 .^{(1)}$

Es una enfermedad genética que afecta a la pigmentación de la piel y suele estar asociada con una gran variedad de alteraciones en ojos, uñas, pelo, dientes, esqueleto, corazón y sistema nervioso central. Se ha observado que la expresión clínica es muy variable, incluso en miembros de una misma familia. Algunas pacientes sólo presentan alteraciones cutáneas, mientras que otras pueden sufrir hasta una severa incapacidad neurológica u oftalmológica. ${ }^{(2)}$ 
Se trata de una enfermedad genética dominante ligada al cromosoma X. La mutación de gran parte del gen NEMO, que incluye exones del 4 al 10, aunque se han descrito otras mutaciones. Las mujeres afectadas sobreviven gracias a la dicigosidad del cromosoma $\mathrm{X}$ y a su inactivación temprana que porta la mutación. ${ }^{(2)}$ Aunque la IP se considera ampliamente letal en hombres, se han reportado más de 120 casos de IP. Esto se puede explicar por 3 mecanismos propuestos: 1-) Cariotipo 47XXY; 2-) Mosaicismo somático en la deleción común NEMO responsable del $80 \%$ de casos IP 46 XX; 3-) Otras mutaciones en el gen NEMO que confieren un fenotipo más leve. ${ }^{(3)}$

El ochenta por ciento de los pacientes con IP llevan mutaciones en el gen NEMO, que codifica el factor nuclear modulador esencial kB (NFkB), el NFkB es crucial para la regulación del factor de necrosis tumoral (TNF) inducido por apoptosis. La reactivación se cree que ocurre cuando hay desencadenantes específicos (posiblemente infección, fiebre o vacunación) que reactivan las vías en las células mutantes residuales. ${ }^{(4)}$

Afecta predominantemente a las mujeres a razón de 35:1 pues en los varones es de carácter letal por las complicaciones intrauterinas que se presentan. Su prevalencia en general es de alrededor de 0,2 en 100.000 niños en base a los datos de 386 pacientes diagnosticados que se informaron en la literatura disponible publicada durante el período 2000-2013.

Incontinentia pigmenti se caracteriza por lesiones cutáneas lineales que comienzan en el nacimiento y la evolución espontánea en cuatro estadios. El estadío 1 es inflamatorio vesiculo-ampolloso con bandas de vesículas epidérmicas llenas de eosinófilos que generalmente están presentes al nacer o en las primeras semanas de vida a los 4-6 meses de edad. En el estadío 2 se aprecian lesiones verrugosas, hiperqueratósicas que pueden aparecer en diferentes áreas distintas al estadío 1, estas se resuelven en 6 meses. El estadío 3 consiste en máculas con hiperpigmentación lineal que aumentan en forma gradual siguiendo las líneas Blaschko; como lo evidenciado en nuestro paciente y un cuarto estadío de hipopigmentación y atrofia. ${ }^{(5)}$

La Biopsia de piel revela un nódulo endoexofítico con invaginaciones del epitelio escamoso queratinizante y un cráter central lleno de queratina, consistente con lesiones similares a queratoacantomas de incontinentia pigmenti. ${ }^{(6)}$

\section{Caso Clínico}

Paciente femenina de 6 años de edad, procedente de Villanueva, Cortés, que es Ilevada a consulta por su madre por cuadro respiratorio alto a la edad de 6 meses, de manera incidental al ser evaluada por el médico observan lesiones de tipo vesiculo-ampolloso y es remitida al servicio de dermatología pediátrica donde en base a los criterios clínicos propuestos por Landy y Donnai se diagnosticó con incontinencia pigmentis.

La madre niega antecedentes personales y familiares relevantes a dicha patología, recibió sus controles prenatales sin anormalidades. Paciente nace vía vaginal, obteniendo un producto femenino, único, vivo cefálico, sin complicaciones, con un APGAR 8 y 9 al 1er y 5to minuto respectivamente, con un peso de 3500 gramos y talla de $50 \mathrm{~cm}$. Se dio en alojamiento conjunto.

Actualmente paciente ha recibido esquema de vacunación completo para la edad según PAI, al examen físico se encuentra por debajo del percentil 10 para el peso y la talla, cabello de aspecto lanudo y alopecia en espiral ondulado, microdontia, rayas hiperpigmentadas que siguen las líneas de Blaschko, que ocurren principalmente en el tronco y extremidades, se pueden observar en la imagen No. 1, 2 y 3. 


\section{Paciente sin deterioro cognitivo, ni alteraciones neurológicas.}

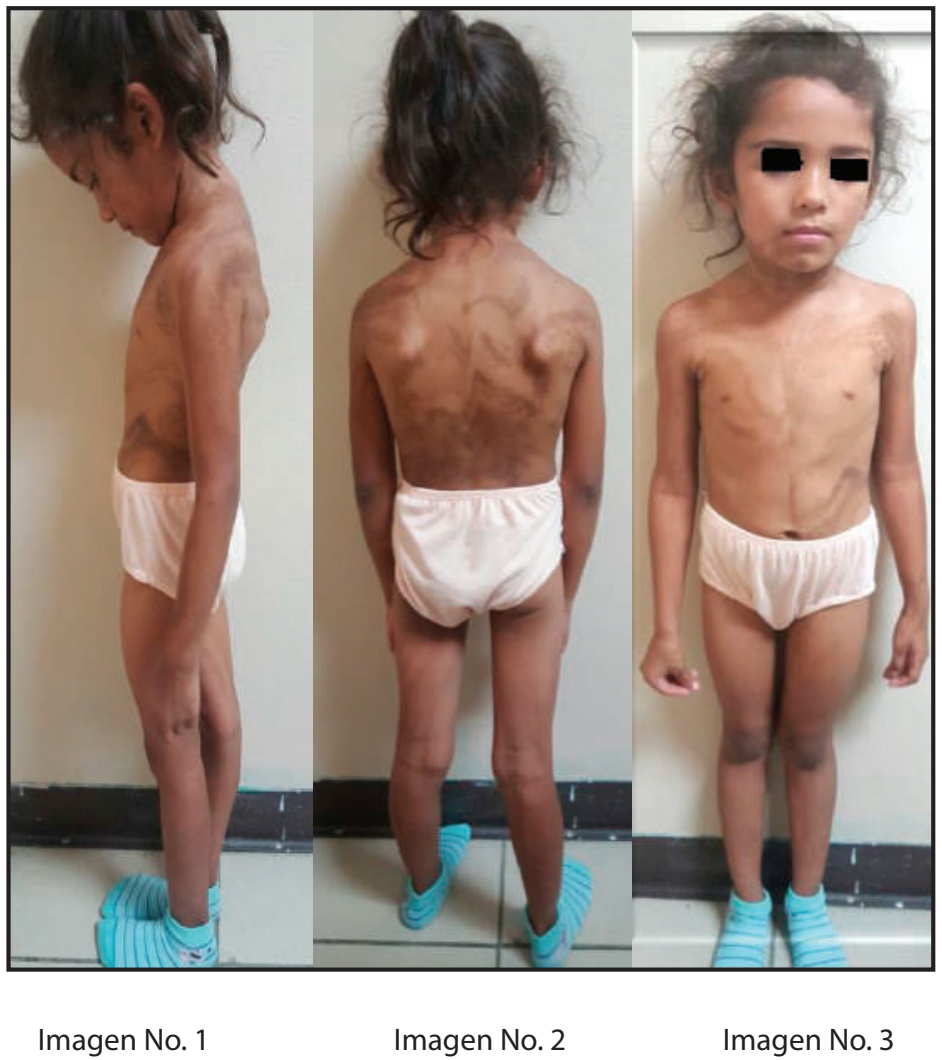

Como el manejo de esta patología es multidisciplinario, se envió al nutricionista para mejorar su estado nutricional y mejorar el desarrollo y crecimiento ya que se ha quedado atrás de acuerdo a los percentiles para la edad, la talla y peso. Al neurólogo para valoración, afortunadamente no ha presentado deterioro neurológico (retraso metal, convulsiones, espasmos infantiles, parálisis espástica, retardo en el desarrollo motor ni microcefalia), oftalmólogo donde a su evaluación no ha presentado estrabismo, ptosis, hipoplasia del iris ni anomalías del desarrollo de los vasos retinianos. Con el servicio de odontología en vista que presenta microdontia se ha dado seguimiento y limpieza bucal, para que a mayor edad se le realizen restauraciones o carillas estéticas.

En las citas de seguimiento con el servicio de dermatología pediátrica a lo largo de estos 6 años ha consistido en mantener la humectación de la piel, la utilización de filtros solares, champu anticaida y el uso de esteroides tópicos en momentos que ha ameritado.

\section{Discusión}

Incontinentia pigmenti (IP) es una genodermatosis rara, dominante ligado a $\mathrm{X}$ causado por mutaciones en IKBKG, inhibidor de la codificación del factor nuclear kappa-B quinasa Subunidad gamma (IKK-C). Dejan células mutantes vulnerables a la apoptosis cuando se expone al factor de necrosis tumoral-alfa. ${ }^{(7)}$

Los hombres afectados por lo general mueren en útero, mientras que las mujeres tienen diversas presentaciones fenotípicas, reflejando el mosaicismo somático a través de la inactivación aleatoria del cormosoma X. ${ }^{(8)}$ 
La prevalencia estimada de IP fue de 0,2 en 100.000 en base a los datos de 386 pacientes diagnosticados que se informaron en la literatura disponible publicada durante el período 2000-2013. ${ }^{(9)}$

Las lesiones cutáneas siguen las líneas de Blaschko, están presentes al nacimiento o poco después y muestran una semiología cutánea muy variada según el estadío en que se observe. Se manifiesta con vesículas, lesiones hiperpigmentadas o verrugosas. No afecta el rostro, las palmas y las plantas. Si bien pueden aparecer sucesivamente, no todos los pacientes padecen todas las etapas y algunas lesiones pueden coexistir en un momento dado.

La incontinencia pigmentaria se asocia con manifestaciones extracutáneas en 50 a $80 \%$ de los casos y afecta predominantemente a tejidos derivados del neuroectodermo y de la cresta neural. Incluye: afectación dentaria en
90\% de pacientes, con alteración del esmalte; defectos esqueléticos, como deformidades craneales, espina bífida o fisura palatina en $40 \%$, y retinianos: desprendimientos, proliferaciones fibrovasculares o cambios pigmentarios.

Las alteraciones neurológicas son frecuentes, en 13 a 50\% pueden expresarse con epilepsia, retraso psicomotor y parálisis espástica o mixta. ${ }^{(10)}$

Como vemos en la paciente el compromiso odontológico es importante caracterizado por la microdoncia y alteraciones en el esmalte de los mismos, considerando también que presenta lesiones cutáneas características de dicha enfermedad como lo describe la literatura. Aún sin afectación a sistema nervioso central, tomando en cuenta los criterios propuestos por Landy y Donnai se realizó el diagnóstico clínico de incontinencia pigmenti. (Ver tabla 1).

Tabla No. 1: Criterios de clasificación de incontinencia pigmenti. Se requiere de al menos un criterio mayor para el diagnóstico y cualquiera de los menores.

\section{Criterios mayores}

Exantema neonatal

- Eritema, vesículas y eosinofilia

\section{Hiperpigmentación típica}

- En tronco

- Líneas de Blaschko

- Hipopigmentación en la adolescencia

\section{Atrofia cutánea}

- Lesiones lineales atróficas

- Lesiones en cuero cabelludo con pérdida de pelo hasta el vértex

\section{Criterios menores}

Anomalías dentales

Alteraciones en retina

Afección de las faneras

- Pelo de aspecto lanudo/ alopecia en espiral - Anomalías de las uñas

Ref. Landy y Donnai. ${ }^{(11)}$ 
A pesar de la raridad de la enfermedad la incontinencia pigmentaria debe siempre ser acordada como diagnóstico diferencial ante recién nacido con alteraciones dermatológicas tipo pápulas y vesículas. La IP es autolimitada y no hay tratamiento específico. El diagnóstico se basa principalmente en el cuadro clínico y en la presentación histológica que es distinta en cada fase evolutiva de la enfermedad. En la fase vesiculoampollosa hay vesículas repletas de eosinofilos; en la fase verrucosa hay hiperqueratosis, disqueratosis, acantosis y papilomatosis; en la fase de hiperpigmentación se evidencia derrame pigmentar con presencia de numerosos melanófagos en la dermis y en la fase de hipopigmentación se encuentra afinamiento de la epidermis y ausencia de anexos.

Los exámenes de neuroimagen pueden ser útiles en los pacientes sintomáticos, pero no son realizados de rutina $y$, por eso, la frecuencia de anormalidades neurológicas en esos pacientes es todavía poco conocida. ${ }^{(12)}$ De requerir evaluar el grado de afectación del sistema nervioso central, la RM es la más recomendada en la evaluación clínica de lactantes con incontinencia pigmentaria. Ellos tienen un patrón característico de las lesiones cerebrales observadas en la RM, mejor reconocidas como imágenes ponderadas por difusión (DWI) e imágenes sensibles a la susceptibilidad (SWI) que permiten la identificación y distinción entre isquemia y lesiones hemorrágicas. ${ }^{(13)}$
El manejo médico-preventivo que ha tenido la paciente a lo largo de sus 6 años de edad ha sido brindado por múltiples servicios, haciendo énfasis en el área dermatológica donde ha presentado la mayor parte de las manifestaciones. El tratamiento incluye lo siguiente: manejo de las ampollas de una manera estándar; Tratamiento tópico para aliviar el malestar. La participación significativa de la piel puede beneficiarse de la gestión dermatológica; Tratamiento de infecciones como para cualquier otra celulitis. Para la neovascularización retiniana que predispone al desprendimiento de retina, crioterapia y fotocoagulación con láser tratamiento estándar para el desprendimiento de retina. Remisión a un neurólogo pediátrico para evaluar si hay microcefalia, convulsiones, espasticidad o déficits focales. La resonancia magnética cerebral en cualquier niño con anomalías neurológicas funcionales o neovascularización de la retina. Referencia a un odontólogo pediatra a los seis meses de edad o cuando los dientes entran en erupción, lo que ocurra primero. Los implantes dentales se han realizado ya a los siete años de edad. Referencia a un patólogo del habla y / o nutricionista pediátrico si la erupción retardada o inadecuada de los dientes primarios interfiere con el masticar y / o el desarrollo del habla. Estimulación adecuada del desarrollo y educación especial como se indica para el retraso del desarrollo. ${ }^{(14)}$ Estas anomalías, especialmente las fonéticas, están relacionadas a problemas de posición y movilidad de la lengua, labios, mejillas y la mandíbula, de las anomalías del arco dental y de la forma de los dientes. Los trastornos del habla pueden considerarse secundarios al síndrome y puede afectar el desarrollo de la comunicación y la interacción de estos niños. En este contexto, destacamos la importancia de un enfoque multidisciplinario. ${ }^{(15)}$ 


\section{REFERENCIAS BIBLIOGRÁFICAS}

1. antiago BE, Brito C, Marte S. Incontinencia pigmentaria: Revisison de casos. Revista Dominicana de Dermatologia. 2015 Enero/ junio; 42(1).

2. Martinez NP, Muñoz IS, Matamoros N. Estudio de continecia pigmenti en España. Nueva muatcio del gen NEMO asociada a inmudeficiencia transitoria. presentacion de caso. Palma de Mallorca: Hospital Son Dureta, Inmunologia; 2012. Report No.: ISBN.

3. Batson R, Brett HK, Diaz LZ. Incontinentia Pigmenti.Insights and images. The Journal of Pediatrics. 2016 May; 176(218).

4. Dupati A, Egbers RG, Helfrich YR, Arbor A, Maryland B. A case of incontinentia pigmenti reacticvation after 12 month inmunizations. JAAD case reports. 2015 Nov; 1(352).

5. Atamari NP, Solorzano Gutierrez S. Incontinentia Pigmanti (Sindrome de Bloch-SulZberger) en un paciente varon. Reporte de caso. Rev. Med Hered. 2015; 26(238-241).

6. Barros B, Helm K, Zaenglein A, Serverling E. Keratoacanthoma-Like Growths of Incontinentia Pigmenti Successfully Treated with Intralesional Methrotexate. Pediatric Dermatology. 2017; 1(2).

7 E. R, Hsu CK, Nanda A, Liu L, H. AA, McGrath JA. Incontinentia Pigmenti in a father and daughter. BritishJournal of Dermatology. 2016; 175(1059-1060).

8. Ali A, Lee A, Swing D, Carrol C, Yosipovitch G. Vaccination as a probable cause of Incontinentia Pigmenti Reactivation. Pediatric Dermatolgy. 2010; 27(1).
9. Rezvan R, Amene Tk. Incontinentia Pigmenti; a Rare Multisystem Disorder: Case Report of a 10- Year-Old Girl. J Dent Shiraz Univ Med Sci. 2016 September; 17(3).

10. Romero A, Tuffiño M, Villacis A, Salazar M. Incontinencia pigmentaria o sindrome de Bloch-Sulzberger. Dermatol Rev Mex. 2014; 58(539-543).

11. Zamora-Chavez A, Escobar-Sanchez A, Sadowinski-Pine $S$, Saucedo-Ramirez OJ, Delgado- Barrera P, Enriquez-Quiñones CG. Incontinencia pigmenti con defecto en la inmunidad celular. Boletin Medico del Hospital Infantil de Mexico. 2015; 72(5).

12. Wacholz Garcia E, Prazeres da Silva A. Relato de caso: Incontiencia pigementar. Residencia Pediatrica. Sociedad Brasileira de Pediatrica. 2015; 5(1).

13. Soltirovska Salomon A, Lichtenbelt K, Frances M C, Casaer A, Dudink J, Anneleen D, et al. Clinical presentation and spectrum of neuroimagen findings in newborn infants with incontinentia pigmenti. Developmental Medicine y Child Neurology. 2016; 10(111).

14. Scheuerle AE, FAAP, FACMG , Ursini MV. Incontinentia Pigmenti. Tesis doctoral. Seattle: University of Washington, Washington; 2015. Report No.: ISSN: 2372-0697.

15. Roce L, Santa Maria fD, Machado Rosa RF, Schermann C, Kszewski A. Stomatognathic and speech alterations are common among children with incontinentia pigmenti. Audiol commun Res. 2015; 20(1). 